\title{
Ballistocardiography Simplified by Recording Microvibration of the Skin Surface of the Head
}

\author{
Toshiyưki Ozaki, Sechiko Sasaki and Katsuro Igarashi \\ The First Department of Physiology, Hirosaki University \\ School of Medicine, Hirosaki
}

Ozakt, T., Sasaki, S. and Igarashi, K. Ballistocardiography Simplified by Recording Microvibration of the Skin Surface of the Head. Tohoku J. exp. Med., 1970,102 (1), 99-100 The microvibration was recorded from the skin surface of the parietal region of the head and compared with the ballistocardiogram simultaneously recorded with a conventional technique. The results suggest the possibility that the microvibration recording from this region may be used in place of a conventional ballistocardiography. — microvibration; head; ballistocardiography.

In the previous paper, Ozaki et al. ${ }^{1}$ reported that there is a close relationship between the dominant waves of the microvibration (MV) of the skin surface above the thenar muscles and the $\mathrm{I}, \mathrm{J}$ and $\mathrm{K}$ wave and $\mathrm{L}, \mathrm{M}$ and $\mathrm{N}$ wave groups of the ballistocardiogram (BCG) in awaking relaxed subjects. It has been known that the head shows rhythmical movements associated with the heart beats, although they are invisible with the naked eye.

The aim of the present study is to determine whether the dominant waves of MV at the skin surface of the parietal region of the head are closely related to the ballistocardiogram recorded by conventional techniques, which have been proved to be useful in studying the cardiac output ${ }^{2}$ and systemic and pulmonary hemodynamies. ${ }^{3}$

The BCG in the longitudinal direction was recorded during breathholding and during resting breathing by use of von Wittern's low frequency table modified by Nihon Kohden Kogyo Co., Tokyo. The sensitivity of the recording system was adjusted so that the load of $300 \mathrm{~g}$ gave a deflection of $30 \mathrm{~mm}$. The MV was recorded simultaneously from the skin surface of the parietal region of the head by means of MV pick up (MT-3T, Nihon Kohden Kogyo Co., Tokyo), which was attached to the skin surface and fixed with a very thin cellophane tape, thereby a perfect contact of the pick up with the skin being ensured. Thus the skin surface of the parietal region was preferred for the reason that this region has little skeletal musculature beneath the skin. In addition, the electro-cardiogram (EKG, limb lead II) was recorded simultaneously to elucidate the relationship of the waves of MV to each phase of cardiac cycle.

Fig. 1 shows the BCG, MV and EKG tracings of a normal subject in a supine position during resting breathing (A) and during breath-holding in the inspiratory phase (B). The dominant waves of MV were found to be composed of two main vibrations corresponding to $H, I, J$ and $K$ wave and $L, M, N$ and $O$ wave groups of the $B C G$ and to $R$ and $T$ waves of the EKG, though the correspondence of $\mathrm{MV}$ to the BCG was more distinct in $\mathrm{H}, \mathrm{I}, \mathrm{J}$ and $K$ wave group than in the $L, M, N$ and $O$ wave one. In addition, the $M V$ obtained from the parietal region was found to be of a slightly lower frequency than that from the regions of the thenar and eyelid. This may probably be due to the paucity of the mus. culature beneath the skin of the parietal region.

In conclusion, dominant waves of the MV showed a pattern very similar to $I, J$ and $\mathrm{K}$ wave group of the $\mathrm{BCG}$, associated with the systole of the cardiac cycle, as shown in Fig.

Received for publication, April 27, 1970. 


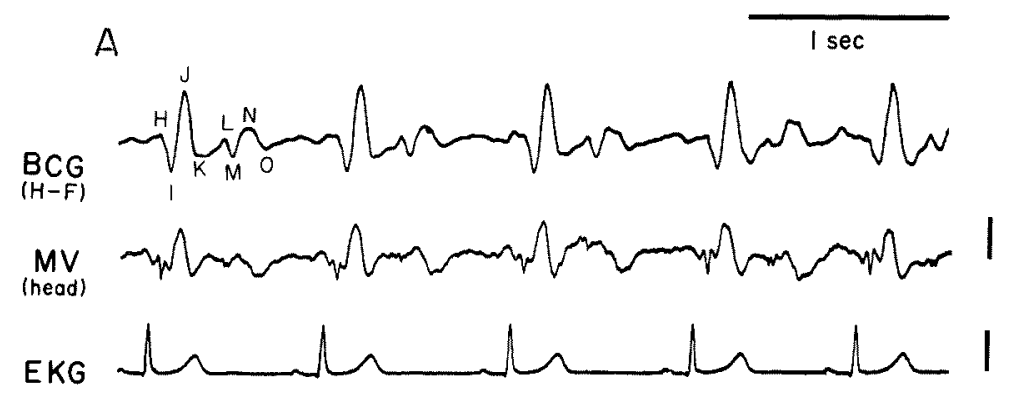

B

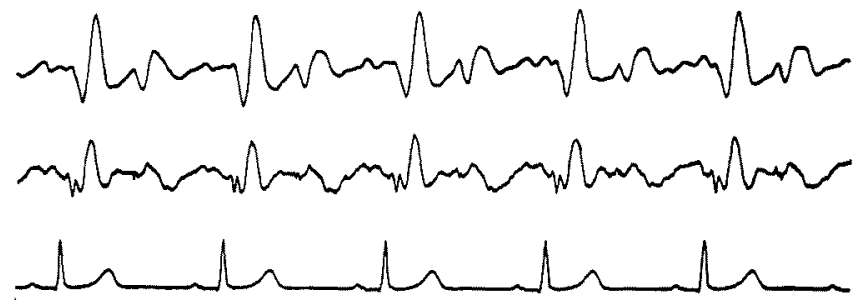

Fig. 1. Ballistocardiogram (BCG), microvibration (MV, head) and electrocardiogram (EKG, Lead II) during rest-breathing (A) and holding of breathing in the inspiratory phase (B). Each calibration in the right side indicates $1 \mathrm{mV}$ in the $\mathrm{MV}$ and EKG, respectively. The calibration of the ballistocardiograph was, on the other hand, so accomodated that the load of $300 \mathrm{~g}$ gave a deflection of $30 \mathrm{~mm}$. Note the appearance of the main components of the MV corresponding to the I, J and K complex of the BCG (H-F) and disappearance in the respiratory fuctuations in the $\mathrm{BCG}$ and $\mathrm{MV}$ during the holding of breathing in the inspiratory phase.

1. The correspondence and similarity between the main components of the MV and the $\mathrm{I}, \mathrm{J}$ and $\mathrm{K}$ wave groups of the BCG were recognized well during the holding of breathing. The main components of the MV increased and decreased in amplitude in the inspiratory and expiratory phases of breathing respectively, where the cardiac output is known to fluctuate physiologically. The present results suggest the possibility that MV of the skin surface of the parietal region of the head may be used as a simple substitute for the conventional ballistocardiography.

\section{Acknowledgment}

We are indebted to Mr. I. Sato for his technical help given us throughout this study.

\section{References}

1) Ozaki, T., Yamamoto M. and Fuziwara, K. On the relationships between minor tremor and ballistocardiogram in man. Acta med. Nagasaki, 1965, 9, 120-134.

2) Sasamoto, H. and Kitada, S. Ballistocardiography (Jap.). Igaku Shoin, Tokyo, 1955.

3) Oike, Y., Onodera, K. and Adachi, M. Ballistocardiographic and electrocardiographic studies in pulmonary hypertension. Proc. 1st world congr. ballistocard. cardiovase. dynamics, Amsterdam, 1965, pp. 313-317.

4) Adachi, M. A study on the relation between ballistocardiogram and pulmonary arterial mean pressure in pulmonary tuberculosis and silicotuberculosis. Jap. circul.J., $1966,30,335-347$. 